\title{
IS THERE A BULGE DISTINCT FROM THE BAR?
}

\author{
KONRAD KUIJKEN \\ Kapteyn Instituut \\ P.O. Box 800, 9700 AV, Groningen, Netherlands \\ and \\ Harvard-Smithsonian Center for Astrophysics \\ 60 Garden Street, Cambridge MA 02138, USA
}

\section{Introduction}

It seems by now well-established that in the central kpc the Galaxy is not axisymmetric (Gerhard 1995, Dwek 1995). The kinematics of many different tracers, as well as the distribution of sources on the sky, all point to an elongated distribution of matter and of the potential, with the long axis of the distortion pointing somewhere in the first Galactic quadrant. It is therefore tempting to reconsider our data concerning this part of the Galaxy in terms of 'the' Bar. But to what extent should we (or is it useful to) distinguish between the bar and the stellar bulge of old?

There are two philosophies that one can adopt. The first is that of the classifier, who divides the Galaxy into as many simple components as seem warranted by the data. This approach is often guided by a preconceived notion of what the components look like: for instance, one might decide a Galaxy contains an outer ring because its disk is not exponential, or a bulge might require a second nuclear component because its profile does not follow the $r^{1 / 4}$ law, etc. This description of the system in terms of basic building blocks is very useful, but it is often not clear in how far one should think of the blocks as different physical entities with their own colours, ages, metallicity, etc., especially since they often overlap considerably in several coordinates (position, velocity, metallicity, age...) A possible second approach is more agnostic: to try to describe the different parts of the Galaxy as they are observed, without necessarily splitting the system into a superposition of components that are familiar or mathematically convenient. From this point of view, one would only talk of different com- 
ponents if their properties were clearly disjoint in one or more observable coordinates.

Clearly the approach to the topic of this review will depend greatly on one's philosophy.

\section{Terminology}

Before we can address the issue of whether there are various components in the central $\mathrm{kpc}$, we need to discuss terminology.

\subsection{WHAT IS A BAR?}

The prerequisite for labelling an object a bar is that it be elongated instead of axisymmetric. Bars were also often said to be as flat as disks. The usual argument is that some $30 \%$ of face-on galaxies are clearly barred, but $30 \%$ of edge-on galaxies do not show an anomalous thickening (Kormendy 1982). However, recent numerical simulations (see section 3 below) have shown that bars are in fact very unlikely to remain thin for very long. Bars are thought to be pattern-rotating, and are kinematically quite hot.

\subsection{WHAT IS A BULGE?}

By a bulge is usually meant a central thickening of a disk (when seen edgeon) or a central brightening of a disk above the exponential Freeman law. Bulges may or may not be related to the metal-poor stellar halo that is seen in the Milky Way. Kinematically, bulges are about as hot as the immediately surrounding disk, and they are often well-described as oblate axisymmetric rotators (Kormendy \& Illingworth 1982). The stellar populations are generally old.

\subsection{DISTINCT IN WHAT SENSE?}

What criteria can we use to decide whether there truly are two distinct components (as opposed to there being a convenient mathematical twocomponent description of the distribution of stars in some coordinates)?

Many physical criteria are possible, and a reasonable fraction of them would have to argue in favour of a separation before it is profitable to distinguish two components.

The following might be reasons in favour of separate components:

- Formation Mechanism With a good understanding of galaxy formation, we might be able to show that bars and bulges are formed by different mechanisms. In that case, it would clearly be important to 
be able to decide on a star by star basis which component to assign objects to.

- Stellar Populations It might be the case that the stellar population nicely splits up into two components. If one of these is characterized as being non-axisymmetric, whereas the other one bulges out above the disk, this would be evidence for two components.

- Shape The spatial structure of a ,alaxy might naturally split into two very different components. This is a dangerous criterion to use, though, since the rotating potential in a barred galaxy can dictate abrupt changes in orbit shapes near resonance radii. For example, the perpendicular 'inner bar' seen in many face-on SB0 galaxies can be caused by an inner Lindblad resonance (accross which the orientation of the orbits changes by $90^{\circ}$ ), and does not imply separate origin or nature of the stars there.

- Kinematics Finally, the velocity distribution might naturally split into two components. However, once again it is important to note that the resonances due to the bar potential may also cause abrupt changes in star and gas kinematics.

Unfortunately, none of the above form very clear-cut practical criteria.

\section{The buckling instability of bars}

It has long been supposed that bars form as a result of an instability in differentially rotating disks (e.g., Sellwood 1981) whereas bulges are a primordial galactic component. In such a scenario, it certainly makes sense to search for ways of separating the two components, in terms of age, chemistry or kinematics. However, it has become clear in the last few years that the picture is not that simple: N-body bars appear to be generically susceptible to a buckling instability which thickens them considerably, and makes them look quite a lot like the box- or peanut-shaped bulges seen in some $30 \%$ of edge-on galaxies (Combes \& Sanders 1981, Combes et al. 1990, Raha et al. 1991). The picture that bars are flat, non-axisymmetric photometric components whereas bulges are vertically extended does not fit in with this theory: so, if the N-body results hold, they imply that a physical bar component must be taken to be vertically extended too.

The fact that the Galaxy has both a boxy bulge and a bar argues for this theory. A numerical simulation of a bar-unstable Milky-Way like model in fact produces a structure which resembles the COBE bulge maps quite well (Sellwood 1993). There are also a few external galaxies known which exhibit both a bar and a boxy bulge: NGC 4442 (Bettoni \& Galletta 1994), which is seen at such an orientation that the bar and the box shape are both visible photometrically, and NGC 5965 and NGC 5746 (Kuijken \& Merrifield 
1994), both edge-on S0 galaxies with boxy bulges whose kinematics provide clear evidence for a barred potential. We are currently developing further the kinematic signature of a bar in edge-on systems, with a view to investigating the link between box-shaped bulges and bars in a larger sample of edge-on galaxies. Without a kinematic test it is difficult to demonstrate the association of the two phenomena, since they are best observed from very different orientations: without kin-matic information, detecting or disproving the existence of a bar in an edge-on boxy-bulge galaxy is about as hard as deciding whether a face-on barred galaxy has a boxy bulge!

It is clear that the buckling instability thoroughly complicates the issue of whether there are separate bulge and bar components, since it implies that bars have significant vertical extent. How then can we usefully distinguish between a lone bar and one with a 'separate' bulge?

\subsection{RECURRING BAR INSTABILITY}

One possible scenario which might lead to distinct components is a recurring bar instability (Hasan, Pfenniger \& Norman 1993). If a disk forms a central bar, then one of the effects is to drive any gas towards the center. Eventually, this gas may form a sufficiently concentrated mass that the orbit structure of the bar is drastically altered: the main orbit family that supports the bar breaks up, as a result of which the bar dissolves. The result of such an episode will then be a fairly axisymmetric thick 'bulge', hotter than the original disk. If the disk now acquires more cold gas, the bar instability may restart, eventually leading to a new buckling bar, and hence a second high-latitude population. Then the term 'bulge' might refer to the material that was formed before the last buckling, and 'bar' to the disk material that formed later and is presently undergoing the bar instability. The bar would then have to be younger than the bulge.

\section{The Milky Way: Two components or not?}

We now turn to a discussion of the available evidence for the Milky Way.

\subsection{STELLAR POPULATIONS}

The bar material after the instability will be found at high latitudes, contaminating any pre-existing 'bulge' material, and unless the stellar populations are very different the result will be a broader spread in age and/or metallicity, not a clear two-component distribution. The data of Tyson \& Rich(1993) show that there is indeed a vertical metallicity gradient on the Galactic minor axis, but it only sets in above a height of $1.5 \mathrm{kpc}$. Above this height the metal-poor stars found at larger radii become quickly dominent. 
Moreover, the distribution of metallicities is well fit by a closed box model. This result therefore argues for a chemically uniform, isolated formation of the bulge material, though a more inhomogeneous evolution followed by mixing (e.g. by a bar instability) may also explain the data.

There is evidence for non-uniformity as well. The late-M giants are very tightly concentrated to the Galactic plane, and to the bulge region (Blanco 1988). This implies that there is at least one thin component in the center of the Galaxy: it therefore has not undergone the buckling instability (yet). Is this evidence for a later burst of star formation? Unfortunately, the kinematics and space distribution of these stars are not sufficiently wellknown to be able to decide if they lie in a bar or not. Since the M-giant luminosity function is a sensitive function of metallicity, it is possible that a moderate metallicity gradient is responsible for this strong observational effect. Nevertheless, it is expected that if the bar buckles, it thickens all populations, so it remains to be seen if the metallicity gradient implied by the $\mathrm{M}$ giant observations is not too strong.

In external galaxies, it is observed that faint and bright bulges have systematically different colour gradients (Balcells \& Peletier 1994). This suggests that the disk exerts some influence in the fainter bulge galaxies, whereas the brighter ones form a more orderly sequence which may indicate a uniform formation scenario. At its most extreme, the disk 'influence' on the fainter bulges may be that the bulge actually forms out of the disk via the bar and buckling instabilities.

\subsection{MORPHOLOGY}

From our vantage point, it is hard to make a convincing case for two superimposed components of different shape in the bulge region. It is clear, though, from perspective effects in near-IR maps, that the distribution of stars above the disk is not axisymmetric, but rather barred (Blitz \& Spergel 1991, Weiland et al. 1994). Of course, a box-shaped bar/bulge superimposed on a primoridal rounder bulge will simply look like a slightly less boxy bulge, unless the radial scale lengths are very different. Only star counts can be used as a way of probing the three-dimensional distribution of stars at all, and the accuracy presently obtainable is barely up to the task. Perhaps the most thorough analysis was made by Weinberg (1992), who analysed IRAS points sources near the Galactic plane: he clearly detects a bar-like distortion, with a central concentration that is probably rounder. It is not clear whether this morphology is usefully described as a two-component structure, though.

Many, but not all, external SB0 galaxies show a perpendicular 'inner bulge', aligned orthogonal to the bar: while these may be consequences 
of the effects of stellar-dynamical resonances, detailed study of this phenomenon is lacking.

\subsection{KINEMATICS}

We would naively expect that a barred population would have a larger velocity dispersion along the bar than perpendicular to it. Therefore, the distributions of radial velocity and of proper motion in longitude in a bar would be expected to be different. A bar seen at $45^{\circ}$ will have identical dispersions in both directions, whereas a more end-on bar (as appears to be favoured by the star count and by the gas kinematics results) should have higher radial velocity dispersion than tangential. The data appear to favour a more or less isotropic velocity distribution, and in fact are well fit by an isotropic oblate axisymmetric rotator model (Kent 1992, Kuijken 1995): stellar dynamical evidence for a bar in the center of the Milky Way are at this point almost non-existent. Only the properties of a sample of stars in Baade's Window with measured three-dimensional velocities (Zhao et al. 1994; Rich 1995, this volume) indicate that the kinematics may not be axisymmetric.

The $\mathrm{OH} / \mathrm{IR}$ stars in the bulge region exhibit quite a clear two-component kinematic structure (Lindqvist et al. 1992), though the sample is not as yet complete. Superimposed on a distribution of stars which broadly follows other kinematic tracers of the disk, in the central $100 \mathrm{pc}$ a fast-rotating group of stars is seen. It has quite a high velocity dispersion too. Some if not most of this may be due to projection of the closed orbits in the barred potential: comparison with the $\mathrm{CO}$ emission over the same region shows that the closed orbits (as identified by Binney et al. 1991) project to velocity widths of $200-300 \mathrm{~km} / \mathrm{s}$. Thus these stars may in reality constitute a very cold component, associated with the molecular gas.

\subsection{CONCLUSION: IS THERE A BULGE AS WELL AS A BAR?}

Few of the data for the Milky Way indicate a strong need for a twocomponent description. All signatures of triaxiality agree on the quadrant in which the long axis lies, plausibly establishing the reality of the bar. The starlight above the disk is elongated in the same direction as the gas orbits close to the plane. There is therefore no need for a two-component description in terms of a more-or-less axisymmetric bulge with a superimposed bar. However, there remains the puzzle of how to fit the (presumably more metal-rich) late- $\mathrm{M}$ giants into the picture: these stars are confined close to the plane, and therefore somehow avoided the buckling instability of the bar which presumably caused the boxy shape of the bulge. 


\section{References}

Balcells, M., \& Peletier, R., 1994, preprint

Bettoni, D. \& Galletta, G. 1994, A\&A, 281, 1

Binney, J., Gerhard, O.E., Stark, A.A., Bally, J. \& Uchida, K.I. 1991, MNRAS, 252, 210

Blanco, V.M., 1988, AJ, 95, 1400

Blitz, L. \& Spergel, D.N., 1991, ApJ, 379, 631

Combes, F., Debbasch, F., Friedli, D. \& Pfenniger, D. 1990, A\&A, 233, 82

Combes, F. \& Sanders, R.H. 1981, A\&A, 96, 164

Dwek, E., 1995. This volume

Gerhard, O.E., 1995. This volume

Hasan, H., Pfenniger, D. \& Norman, C., 1993, ApJ, 409, 91

Kent, S.M., 1992, ApJ, 387

Kormendy, J. \& Illingworth, G. 1982, ApJ, 256, 460

Kormendy, J., 1982 in Morphology and Dynamics of Galaxies, ed. L. Martinet \& M. Mayor, (Geneva Observatory), 237

Kuijken, K., 1995, preprint

Kujken, K. \& Merrifield, M.R., 1994, ApJL, submitted

Lindqvist, M., Winnberg, A., Habing, H.J. \& Matthews, H.E. 1992, A\&AS, 92, 43.

Raha, N., Sellwood, J.A., James, R.A. \& Kahn, F.D. 1991, Nature, 352, 411

Rich R.M., 1995, this volume

Sellwood, J., 1981, A\&A 99, 362

Sellwood, J., 1993, in Galactic Bulges, IAU Symposium 154, eds. H. Dejonghe \& H.J. Habing, 391

Tyson, N.D. \& Rich, R.M., 1993, in Galactic Bulges, IAU Symposium 154, eds. H. Dejonghe \& H.J. Habing, 333

Weiland, J.L., et al., 1994, ApJ 425, L81

Weinberg, M.D., 1992, ApJ, 384, 81

Zhao, Spergel, D.N. \& Rich, R.M., 1994, preprint

\section{DISCUSSION}

M. Balcells: When comparing the kinematic predictions of the various Milky Way bulge models (bar, axisymmetric structure) you did not mention extinction. Shouldn't we always extinct our models before comparing to optical tracers? And, can we make extinction play in our favour to further discriminate among models? E.g., if the back side of the bar is dimmer than the near side, we may observe non-zero mean velocity at the bar center.

M. Weinberg: I am worried that the inference of a perpendicular bulge component based on the figure from my 1992 paper (ApJ 384, 81) may be an artefact of the 2 Jy cutoff. Although a $1 \mathrm{Jy}$ cutoff is certainly incomplete, it does not show such a pronounced perpendicular component. 